\title{
Filling of three-dimensional space by two-dimensional sheet growth
}

\author{
Merlin A. Etzold, ${ }^{1, *}$ Peter J. McDonald, ${ }^{2}, \dagger$ David A. Faux, ${ }^{2,}+$ and Alexander F. Routh ${ }^{1, \S}$ \\ ${ }^{1}$ BP-Institute, University of Cambridge, Madingley Road, Cambridge, CB3 OEZ, United Kingdom \\ ${ }^{2}$ Department of Physics, University of Surrey, Guildford, Surrey, GU2 7XH, United Kingdom
}

(Dated: September 9, 2015)

\begin{abstract}
Models of three-dimensional space filling based on growth of two-dimensional sheets are proposed. Beginning from planar Eden-style growth of sheets, additional growth modes are introduced. These enable the sheets to form layered or disordered structures. The growth modes can also be combined. An off-lattice kinetic Monte-Carlo-based computer algorithm is presented and used to study the kinetics of the new models and the resulting structures. For the first time, it is possible to study space filling by two-dimensional growth in a three-dimensional domain with arbitrarily-oriented sheets; the results agree with previously-published models where the sheets are only able to grow in a limited set of directions. The introduction of a bifurcation mechanism gives rise to complex disordered structures that are of interest as model structures for the meso-structure of calciumsilicate-hydrate in hardened cement paste.
\end{abstract}

\section{INTRODUCTION}

Space filling by growing structures originating from one or more nuclei is a class of problem that was studied first by metallurgists. A solidifying metal can be described as a volume which is progressively consumed by growing crystals. The work presented in this article was triggered by the problem of the description of calciumsilicate-hydrate formation and structure in cement [1-4]. This material is widely described as an amorphous sheet structure [5]. Well-characterised model cement mesostructures are necessary for understanding leading to the development of more durable and environmentallyfriendly cement-based materials [4]. The problem of sheet structures is, however, not limited to cement. Researchers studying enhanced oil recovery from shales [6] and transport processes of radioactive contaminants in clays [7] need suitable model structures. Model polycrystalline structures are also required to aid the understanding of gas flows in catalysts [8] and, of course, crystal growth remains of wide interest in many disciplines of science [9]. The generalised problem includes any $d_{g^{-}}$ dimensional growth in a $\left(d_{g}+1\right)$-dimensional space. For example, this problem has been studied in the context of orientational linear epitaxy [10] and fragmentation [11].

In this paper, we explore the formation of sheet structures from a kinetic aspect: in Sec. II, we define a series of sheet-growth models, leading to different types of structure. The kinetics of formation, leading to these resultant structures are investigated.

The most popular model for space filling is the Kolmogorov-Johnson-Mehl-Avrami (KJMA) model [1214]. It assumes growth of isotropic particles in an infinite domain originating either from continuous nucleation or

\footnotetext{
* merlinaetzold@cantab.net

$\dagger$ p.mcdonald@surrey.ac.uk

$\ddagger$ d.faux@surrey.ac.uk

$\S$ alex@bpi.cam.ac.uk
}

a fixed number of initial nuclei. Its central result reads

$$
\varphi(t)=1-\exp \left(-k t^{n}\right),
$$

where the volume fraction of solidified material at time $t$ is denoted by $\varphi(t)$, and $n$ is the growth exponent. The growth exponent is equal to $d_{S}$, the dimension of growth, if nuclei are initially present (site saturation), or $d_{S}+1$ if nucleation is linear in time. $k$ is a constant, collecting a range of subsidiary constants invoked during the derivation, including the nucleation rate or the number of initial nuclei, and the radial growth rate of the individual crystals. The properties and various extensions of this model are an active area of research. Particular interest has been attracted by the statistical properties of the structures formed [15-19]. Various approaches have been investigated to go beyond the limitations of the original model. As Shepilov and Baik point out, the KJMA equation is not valid for diffusion type kinetics, in finite domains for anisotropic growth with arbitrary orientation [20], since mutual blocking can occur otherwise. Extensions towards diffusion-type kinetics, for example, have been proposed by Alekseechkin and Tomellini and Fanfoni $[21,22]$. Furthermore, KJMA-style growth in finite domains is a field of active research [23, 24].

The problem studied in this paper is an extreme case of anisotropic growth. Similar to the present work, anisotropic growth is typically studied numerically using ellipsoids or their two-dimensional (2D) equivalents as growing objects. Specifically, anisotropic growing objects can block each other, or at least delay growth as a blocked shape grows around the obstruction. In Shepilov's and Baik's work, the interaction between ellipsoids was treated explicitly for up to two subsequent blockings [20]. Pusztai and Gránásy investigated the growth of ellipses in two-dimensional space. The interaction between the growing ellipses, such as mutual exclusion, was treated by mapping the ellipses on a cubic lattice [25]. A similar approach was chosen by Godiksen et al., to study the growth of ellipsoids in three-dimensional (3D) space [26]. These authors observed that for mild anisotropy of the growing shapes, the KJMA equation still holds. 
Two-dimensional growth within a 3D space was studied by Kooi [27]. This author proposed a lattice-based Monte-Carlo model able to describe the growth of twodimensional sheets within 3D domains. Kooi also studied the similar situation for one-dimensional (1D) objects (needles) in 2D space. The lattice-based simulation, however, restricts the orientation between the growing objects to those permitted by the lattice. In the case of continuous nucleation, growth initially follows the KJMA kinetics. Later, the growth exponent decreases, indicating the onset of blocking of growth from different nuclei. This causes the growth exponent to decrease towards a minimum, followed by an increase towards a limiting value due to the ongoing nucleation in the void space between sheets. Kooi refers to this second stage of growth as the blocking regime [27]. The characteristic time $t^{*}$ for the onset of blocking of $2 \mathrm{D}$ growth in $3 \mathrm{D}$ is

$$
t^{*} \propto \frac{1}{k_{N}^{1 / 4} \dot{r}^{3 / 4}},
$$

where $k_{N}$ is the nucleation rate (events per unit time), and $\dot{r}$ is the radial growth rate of the sheets. Kooi also showed that, in the blocking regime, the volume of grown solids is given as

$$
V\left(t>t^{*}\right)=V\left(t^{*}\right)+Z h\left[k_{N}\left(t-t^{*}\right)\right]^{\frac{1}{3}},
$$

where $V\left(t^{*}\right)$ is the volume grown to time $t^{*}, Z$ is a constant, and $h$ is the sheet thickness. As $t$ becomes large, the growth exponent is predicted to approach $1 / 3$. Equations 2 and 3 can be derived by considering the mean distance between the seed points and combining these with the maximum characteristic size of the resultant sheets. Kooi also presents comprehensive kinetic equations, which, as he points out, are only valid for the specific geometry (namely the constraint in the sheet orientation) considered in their simulations [27].

The work presented in this paper goes beyond Kooi's work and addresses the problem of off-lattice 2D growth of sheet-like structures within 3D space with opportunity for different defects within the crystallites. Specifically, space filling is achieved by growing disordered sheet structures. In many systems, such as a growing crystal, it is reasonable to assume that various defect mechanisms affect the growth of pure sheets [5]. We propose a range of mechanisms as to how defects can introduce disorder in the sheet structures, including "within sheet" and "next-layer" disorder. We analyse them individually and the effects of their combination under different scenarios. The numerical method presented is able to produce arbitrarily-oriented sheets, so a randomly-oriented version of Kooi's model can be studied.

Models of space filling are typically analysed by assuming that growth follows Eq. 1, which can be rewritten as $\ln (-\ln (1-\varphi(t))=\ln k+n \ln (t)$. The corresponding plot is called an Avrami plot and it enables the growth exponent to be determined. The application of this analysis to data from a KJMA scenario leads to a straight line. The inner logarithm represents the interaction between the growing shapes in Avrami's original derivation [14]. In an anisotropic growth scenario, one expects that the shapes impinge earlier than in the case of growing (hyper) spheres and thereby slow growth, changing the growth exponent.

This paper is organised as follows. The growth model is developed in modular fashion in the next section with its implementation via numerical simulation described in Sec. III. The results for five different growth models are presented and discussed in Sec. IV and the key conclusions are drawn in Sec. V.

\section{THE SHEET GROWTH MODEL}

\section{A. Model elements}

Sheets are discretised as triangular prisms with side length $L$ and height $h$. These segments can take arbitrary positions and orientations in the simulation domain. The base model describes growth of sheets in space and consists of the following model elements:

1. Two segments may not overlap. Any point in space can only belong to a single segment.

2. Nucleation is modelled by randomly placing a segment in the domain. Thus, nuclei have finite size and an orientation.

3. Sheet growth is modelled by the $2 \mathrm{D}$ Eden model [28]. For each growth event, a random site along the perimeter of a sheet is chosen and a new segment is inserted. These events occur with a rate $k_{S}$.

These elements yield a model of $2 \mathrm{D}$ sheet growth in $3 \mathrm{D}$ space. We assume that pure sheet growth is unlikely to occur in a physical system; defects or the formation of layers may also be present. Therefore, we also define the following growth mechanisms. In all cases, we assume that the other rate constants are much lower than the rate constant for planar sheet growth. The elements below are referred to as additional growth modes.

4. At each site available for planar growth, a process can occur where a new segment is tilted by an angle $\gamma_{B}$ as shown in Fig 1c. This occurs with a rate $k_{B}$ and is referred to as bifurcation.

5. A new segment may be placed parallel to an existing segment at a distance $d$ (above or below) the existing segment. This is termed layering, and the facet of a segment that layering originates from is referred to as a layering site, as illustrated in Fig. $1 \mathrm{~d}$.

6. Tilted layering occurs with a rate $k_{T}$. This process is similar to layering, but the new segment is rotated by an angle $\gamma_{T}$ around an arbitrary edge. This is illustrated in Fig. 1e.

These elements can be combined into a range of different growth models, which are outlined in Sec. II B. It should be noted that these concepts would equally work with other symmetric shapes such as square prisms. 
Note that the growth mechanisms, particularly the Eden growth mechanism of sheets, is phenomenological and could have a wide range of physical origins, which will be discussed in some detail in Section V.
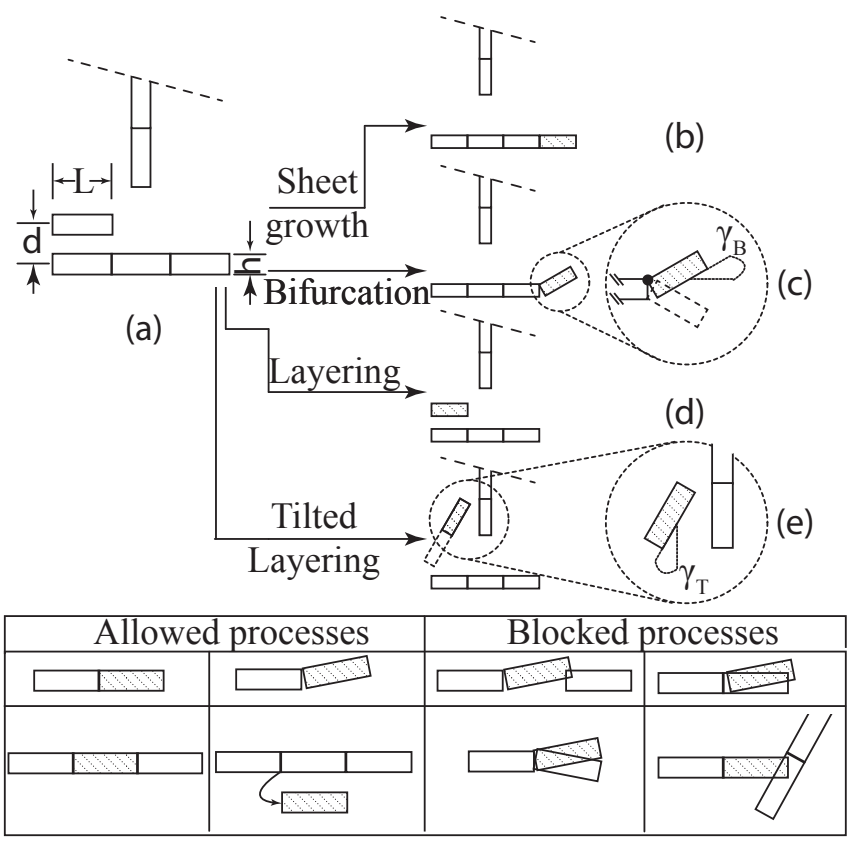

FIG. 1. Two-dimensional illustration of the key elements and the growth steps. The new segment is always indicated. (a) shows an example with two sheets about to impinge with perpendicular angle and the definition of sheet thickness $h$, interlayer space $d$ and segment side length $L$. (b) shows a planar growth step extending the horizontal sheet, (c) a bifurcation step. The enlargement defines the bifurcation angle $\gamma_{B}$ and shows the alternative bifurcation process with dotted lines. (d) shows a layering step and (e) a tilted layering step. The enlargement defines the tilt angle $\gamma_{T}$ and the rotation axis relative to a layering step occurring at the same position (dotted rectangle). The filled circles denote the rotation axis. Below: table of allowed and blocked configurations in a $2 \mathrm{D}$ illustration.

\section{B. Combinations of model elements}

The model elements can be combined with sheet growth to produce a range of different growth models. These models are presented as a hierarchy shown in Fig. 2. The first column describes growth from a single nucleation site and the second column extends to multiple nucleation sites or continuous nucleation. The models are assigned to the different rows to represent their increasing complexity in terms of the number of free growth parameters.

The start of this hierarchy is the 2D Eden model in Fig. 2a. If this model is extended to grow from multiple randomly oriented nuclei, Fig. 2b, 3D space is filled by an open structure, which densifies in the continuous nucleation case. The free parameter is the ratio between

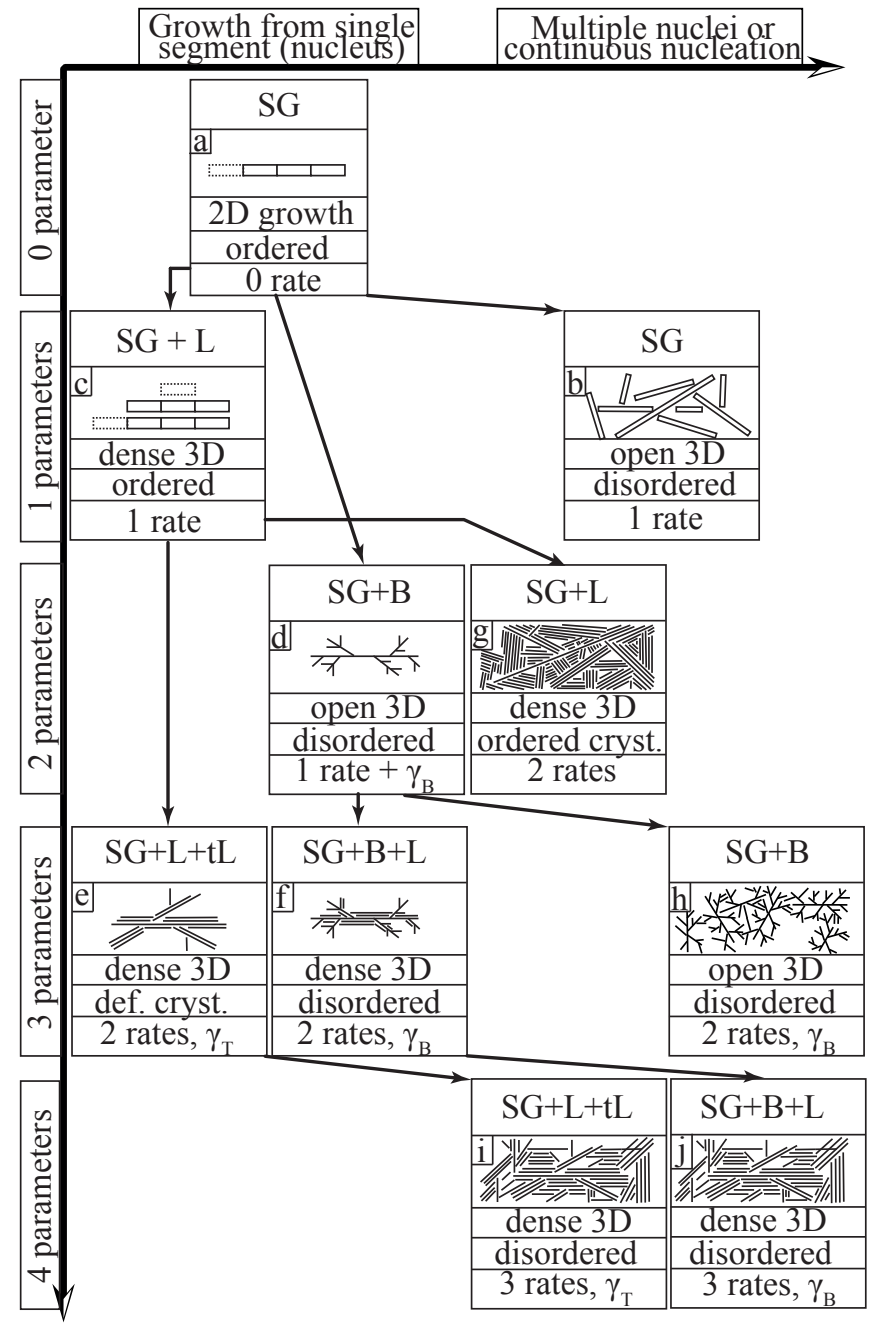

FIG. 2. Overview of the sheet growth models with 2D illustrations. Starting from the Eden model (sheet growth (SG)), gradual addition of complexity via a layering (L), tilted layering (tL) or bifurcation (B) mechanism leads to models forming complex clusters. Introducing a nucleation model or a finite domain turns the growth models into models of space filling.

nucleation and sheet growth rates, $k_{N} / k_{S}$. Since the sheets can take random orientations, this is an extension of Kooi's model [27].

The 2D Eden model can also be extended by the layering step, leading to the growth model shown in Fig. $2 \mathrm{c}$, which has the ratio between layering rate and sheet growth rate, $k_{L} / k_{S}$, as the single free parameter. This growth model leads to perfect and dense sheet crystals filling 3D space. Fig. 2g shows space filled with sheet crystals leading to $3 \mathrm{D}$ polycrystalline structures. The ratio $k_{L} / k_{S}$ controls the anisotropy of the resultant crystallites. A further extension can be made with the introduction of tilted layering, as shown in Fig. 2e. This introduces the new geometric parameter $\gamma_{T}$, and has two further free parameters: the ratio $k_{L} / k_{S}$ and the ratio between tilted layering rate and sheet growth rate, 
$k_{T} / k_{S}$. In the continuous nucleation scenario (Fig. 2i), this model leads to dense polycrystalline structures, but the average number of repeating layers will be lower than in the scenario with layering only (Fig. $2 \mathrm{~g}$ ). We have previously explored this particular model suggesting a first sheet growth model for cement [1].

The extension of the 2D Eden model by the addition of the bifurcation mechanism is shown in Fig. 2d. The bifurcation mechanism allows occasional segments to change their growth direction. The resultant structures are expected to be open and complex. This model has two free parameters, the ratio between bifurcation and sheet growth rates $k_{B} / k_{S}$ and the bifurcation angle $\gamma_{B}$. The extension of this model towards 3D space filling leads to a model with three free parameters by adding $k_{N} / k_{S}$ (Fig. 2h). A further extension of the model shown in Fig. $2 \mathrm{~d}$ by adding the layering mechanism is illustrated in Fig. 2f with the 3D space-filling extension presented in Fig. 2j.

\section{Theory of sheet growth}

In this section, we present theory to describe how structural shapes originating from a single nucleus develop in time.

\section{Two-dimensional growth}

Pure 2D growth of a single sheet leads to a 2D object. It has a characteristic size $r$, which could be the radius of a circle or the side length of a square. The surface area of the object is denoted $A$. The characteristic size expands by $\mathrm{d} r$ during the time interval $\mathrm{d} t$. If the shape is sufficiently large, $\mathrm{d} r$ can be approximated by the height of a base triangle, which makes up the sheet. This is sketched in Fig. 3.

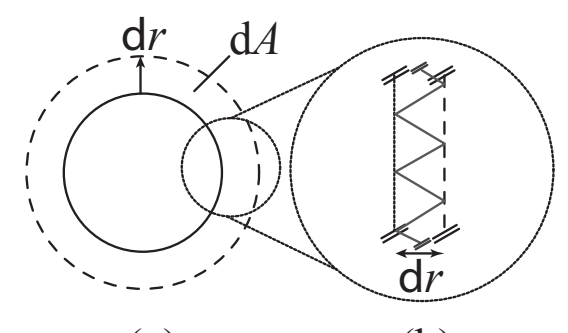

(a)

(b)

FIG. 3. The radius of a circular object expands by $d r$ in a time interval $d t$ resulting in an increase in area $d A$.

The number of equilateral triangular segments with side length $L$ at time $t, N(t)$, changes each time step such that:

$$
\frac{\mathrm{d} N}{\mathrm{~d} t} \approx \frac{1}{A_{\triangle}} \frac{\mathrm{d} A}{\mathrm{~d} t}=2 \frac{C}{A_{\triangle}} r \frac{\mathrm{d} r}{\mathrm{~d} t}
$$

where the constant $C$ relates the characteristic size to the area, such that $A=C r^{2}$. Here, $A_{\triangle}=\frac{\sqrt{3}}{4} L^{2}$ is the surface area of an equilateral triangle with side length $L$. It is reasonable to assume that the radial growth rate is proportional to one of the characteristic sizes of the triangle, thus:

$$
\frac{\mathrm{d} r}{\mathrm{~d} t} \sim k_{S} L
$$

The proportionality constant to be in the order of one. If one further assumes circular growth and sets $C=\pi$ as in a circle and integrates, one obtains

$$
N(t) \sim \frac{4}{\sqrt{3}} \pi k_{S}^{2} t^{2} .
$$

This equation describes growth of a single sheet in the limit of an infinite-sized structure.

An alternative way to derive the same functional form for the increase starts with the well-known property of the Eden model that the number of perimeter segments $P$ scales as the $\sqrt{N}=\sqrt{A} / \sqrt{A_{\triangle}}[28,29]$. Since the number of inserted triangles per unit time is proportional to the number of perimeter segments, one can assume that the rate of increase in number of triangles is proportional to the perimeter of the sheet, hence

$$
\frac{d N}{d t}=k r
$$

where the rate constant $k$ has dimensions of inverse length inverse time which is different from the constant $k_{s}$ which has dimension inverse time. Recognising that the number of triangles in the sheet is related to the sheet area by $N=\pi r^{2} / A_{\triangle}$ one arrive at the relation

$$
N(t)=\frac{k^{2} A_{\triangle} t^{2}}{4 \pi}
$$

Which is the same functional form as equation (6) and one can recognise the relation $k \sim \pi \sqrt{\frac{64}{3}} \frac{k_{s}}{L}$.

\section{Evolution of a bifurcation cluster}

In Fig. 4a, an early bifurcation cluster is sketched. It consists of a planar parent sheet, which is referred to as zeroth generation. On its perimeter sites, bifurcation events can occur which give rise to new sheets, which grow in space as also illustrated in Fig. 4b. These sheets are referred to as first generation. At the perimeter of the first generation sheets, further bifurcation events can occur, the resultant sheets are then the second generation sheets and so forth.

The sheets of the first and higher generations belonging to the same bifurcation cluster are able to impinge on each other as shown in Fig. $4 a$ and $b$.

In $3 \mathrm{D}$, it is clear that sheets from the first generation can impinge on the zeroth generation, and on other first 
generation sheets, though, this is not the general case in $2 \mathrm{D}$ growth as illustrated in Fig. 4c. As Fig. 4a indicates, each bifurcation site effectively serves as the origin of an approximately half-circular sheet (or more if it rounds and extends beneath the parent sheet).

More generally, a child sheet can impinge on the parent and on another child of that parent. However, there is a subtle difference between child-parent and child-child impingement. A child sheet impinging on its parent does not prevent the child sheet extending semi-infinitely, whereas a child impinging on a sibling, or indeed, a sheet of another generation more generally, cannot necessarily grow semi-infinitely.

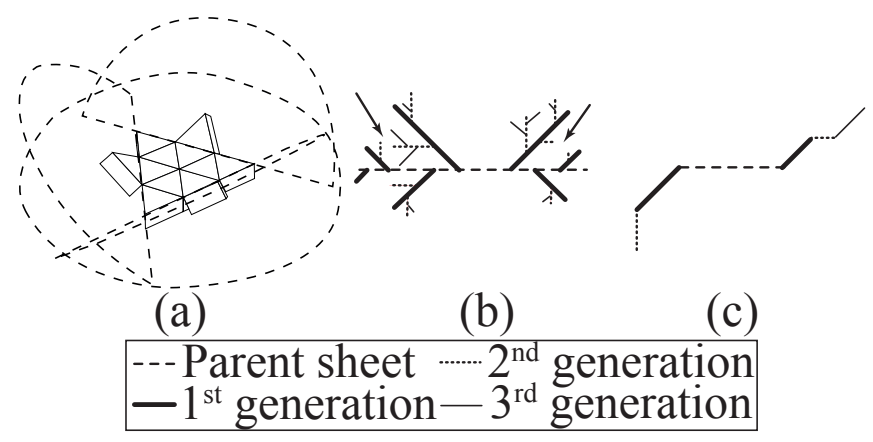

FIG. 4. (a) Three-dimensional illustration of a small sheet with three bifurcation events, which are all rotated upwards for the clarity of the sketch. The dotted circle marks the plane of the primary sheet, the three dotted half-circles indicate the plane of the sheets formed by bifurcation. They eventually intersect. (b) $2 \mathrm{D}$ illustration of a cross section through the tree-like structure generated by sheet growth with bifurcation. At at least two sites (arrows) internal impingement is imminent. (c) Illustration of a bifurcation-like process in $2 \mathrm{D}$ for $1 \mathrm{D}$ growth.

Since impingement of a child with a parent does not limit the growth of a sheet, Eq. 6 should hold in the absence of multiple impingements between siblings and other higher generation sheets. Such multiple impingements will eventually deny infinite space to a growing sheet.

For simplicity, let us consider a growing sheet in space; bifurcation events are possible at the perimeter of the sheet, effectively leading to new sheets, similar to a nucleation event. For the moment, it is assumed that, at the new sheets, no further bifurcation events are possible. The number of triangular segments in a sheet at time $t$ that was nucleated at $t^{\prime}$ is denoted as $N\left(t, t^{\prime}\right)$. The length of the perimeter of the primary sheet is proportional to its characteristic size, so one has $L_{P} \sim\left(t-t^{\prime}\right)$. Since bifurcation can occur at each perimeter segment, the number of bifurcation events is $\beta\left(t-t^{\prime}\right)$. The constant $\beta$ contains the necessary proportionality factors and the bifurcation rate. Combining this with $N\left(t, t^{\prime}\right)$ leads to the number of segments in a first-generation-only bifurcation cluster

$$
N\left(t, t^{\prime}\right)+\int_{t^{\prime}}^{t} \beta\left(t^{\prime \prime}-t^{\prime}\right) N\left(t, t^{\prime \prime}\right) d t^{\prime \prime} .
$$

If bifurcation is also possible on those sheets which were formed by bifurcation themselves, a recursive expression can be obtained if internal intersection between the sheets grown from the same cluster is ignored. Internal impingement is inevitable and occurs at some point and leads to a decrease of the growth exponent. The aforementioned recursive expression can be evaluated, if one assumes that only $J$ generations of bifurcation events occur. This leads to

$$
N\left(t, t^{\prime}\right) \sim t^{2+2 J}
$$

still assuming that the sheets formed by bifurcation do not impinge on each other internally.

The growth exponent is expected to decrease as soon as internal impingement occurs. At least one generation of sheets can be formed by bifurcation before these impinge on each other, and so $\min (J)=1$. Thus, the lower bound for the growth exponent for sheet growth with bifurcation is 4 in the early stages of growth. Impingement can either occur in the plane of the first sheet (see Fig. 4a) or, as shown in Fig. 4b, between sheets of the second generation. Therefore, it is unlikely that more than two generations of sheets form by bifurcation at any time, thus, $\max (J)=2$. This leads to the upper bound for the growth exponent for sheet growth and bifurcation of 6 in the early stages of growth.

Leyvraz points out that, in any growth model, only those sites which are located on the surface of the convex hull of a cluster are able to sustain growth [30]. Therefore, the growth exponent is expected to decrease towards three for very large clusters.

\section{NUMERICAL SIMULATION}

In order to simulate sheet growth, a computer algorithm must be able to describe the positions of the sheets in space and simulate the growth as a function of time. The segments are kept in a framework developed for this purpose similar to the CGAL library [31], and evolution of time is tracked via a kinetic Monte Carlo (KMC, or alternatively also dynamic Monte Carlo) scheme. This is an advance from previous lattice-based codes, since these programs were unable to handle randomly-oriented sheets.

In each iteration, a list of all processes to be attempted is formed. Based on this list, a rate line is computed. Its inverse length determines the length of the time step associated with the iteration. A random point on the rate line is chosen, and the process associated with this point is carried out. The algorithm was implemented in Java and can handle domain sizes of up to $(400 \cdot L)^{3}$ with ca. $10^{8}$ individual triangular segments.

The KMC scheme uses an algorithm which was first proposed for simulating Ising spin systems [32], coupled systems of chemical reaction [33] and crystal growth [34]. These applications and further details are described in 
reviews by Chatterjee and Vlachos, Voter, and Novotny [35-37]

\section{A. Verification: single sheet growth}

In this section, the numerical simulation is verified by simulating a 2D Eden model. The radius of the domain was $2500 \mathrm{~L}$; growth was terminated as soon as the growing sheet touched the domain boundary, so as to exclude any boundary effects. Following Eq. 5 and 6 , data are fitted with the power laws $N(t)=A_{N} t^{n}$ and $r(t)=A_{r} t^{n_{r}}$. The radius of the sheet is calculated from the radius of gyration, $r_{g}$, as $r=\sqrt{2} r_{g}$. For the remainder of this paper, the radius of gyration is defined via the centroids of the segments.
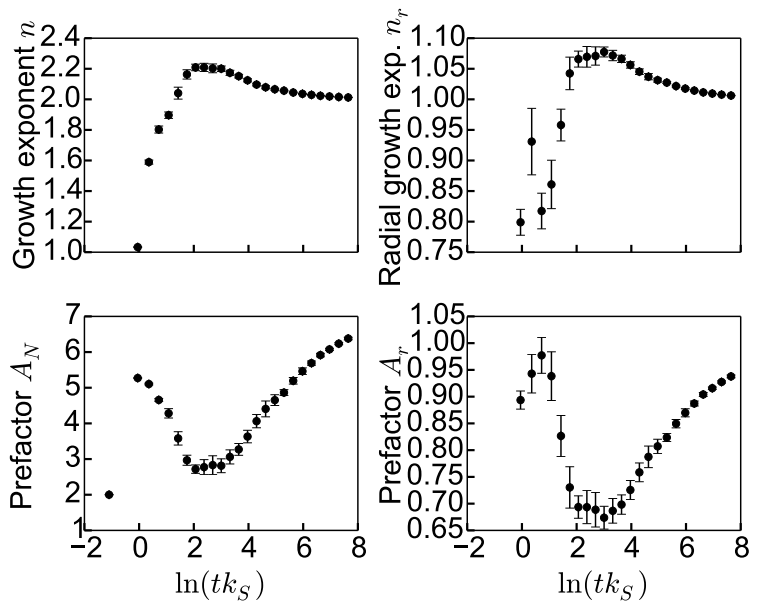

FIG. 5. Unhindered growth of a single sheet in space. The graphs show the growth exponent, the radial growth exponent, the fitted prefactor to the power law $A_{N}$ and the fitted prefactor $A_{r}$. The error bars represent the standard error of the mean for nine repetitions.

In the top-row of Fig. 5 the growth exponent $n$ and the radial growth exponent $n_{r}$ are plotted against natural logarithmic reduced time. The growth exponent increases from an initial value of 0.5 towards a maximum of about 2.3 , and subsequently converges around 2 . The radial growth exponent follows a similar trend. It starts at about 0.85 , before increasing towards 1.05 . Notably, the error bars during this increase, calculated from the standard error of 9 repetitions, are comparatively large. This reflects the statistical variability in the early sheet shape. As time progresses further, the error decreases considerably. In the long time limit, the curve approaches a value of 1 . Therefore, the time-evolution of the clusters scales as predicted by Eq. 5 and 6 .

The bottom-row of Fig. 5 shows the prefactors of the power laws as a function of logarithmic reduced time. These were calculated using the growth and radial growth exponents found at the corresponding times. The prefactor $A_{N}$ seems to converge to a value around seven, whilst
TABLE I. Parameters for the growth models presented in this paper. D. denotes the domain size. If it is written with a dot $(\cdot)$ a cylindrical geometry was used, otherwise a cubic domain was employed. In the first case, the growth was stopped as soon as the first segment intersected with the domain boundary, in the second, the domain was periodic. The column labelled $\mathrm{R}$ is the number of repetitions.

\begin{tabular}{|c|c|c|c|c|c|c|c|c|}
\hline Sec. & D. $/ L^{3}$ & $h / L$ & $d / L$ & $k_{L} / k_{S}$ & $k_{B} / k_{S}$ & $\gamma_{B}\left[^{\circ}\right]$ & $k_{N} L^{3} / k_{S}$ & $\mathrm{R}$ \\
\hline III A & $400^{3}$ & 0.1 & - & - & - & - & - & $\overline{9}$ \\
\hline \multirow{3}{*}{ IV C } & \multirow{3}{*}{$400^{3}$} & \multirow{3}{*}{0.85} & \multirow{3}{*}{ - } & \multirow{3}{*}{ - } & & & $10^{-6}$ & \\
\hline & & & & & \multirow{2}{*}{ - } & \multirow{2}{*}{ - } & $10^{-7}$ & 4 \\
\hline & & & & & & & $10^{-8}$ & 4 \\
\hline \multirow{4}{*}{ IV A } & $500^{3}$ & \multirow{4}{*}{0.5} & \multirow{4}{*}{0.5} & 10 & \multirow{4}{*}{-} & \multirow{4}{*}{-} & \multirow{4}{*}{-} & \multirow{4}{*}{6} \\
\hline & $400^{2} \cdot 600$ & & & 1 & & & & \\
\hline & $800^{2} \cdot 200$ & & & $10^{-3}$ & & & & \\
\hline & $2000^{2} \cdot 100$ & & & $10^{-7}$ & & & & \\
\hline \multirow{5}{*}{ IV D } & \multirow{5}{*}{$270^{3}$} & \multirow{5}{*}{0.5} & \multirow{5}{*}{0.5} & 1 & \multirow{5}{*}{-} & \multirow{5}{*}{-} & \multirow{5}{*}{$10^{-6}$} & \multirow{5}{*}{4} \\
\hline & & & & 10 & & & & \\
\hline & & & & $10^{-3}$ & & & & \\
\hline & & & & $10^{-4}$ & & & & \\
\hline & & & & $10^{-7}$ & & & & \\
\hline \multirow{6}{*}{ IV B } & \multirow{6}{*}{$200^{2} \cdot 200$} & \multirow{6}{*}{0.1} & \multirow{6}{*}{-} & \multirow{6}{*}{ - } & $10^{-2}$ & 11 & \multirow{6}{*}{ - } & \multirow{6}{*}{6} \\
\hline & & & & & $10^{-3}$ & 11 & & \\
\hline & & & & & $10^{-2}$ & 45 & & \\
\hline & & & & & $10^{-3}$ & 45 & & \\
\hline & & & & & $10^{-2}$ & 85 & & \\
\hline & & & & & $10^{-3}$ & 85 & & \\
\hline \multirow{3}{*}{ IV E } & \multirow{3}{*}{$350^{3}$} & \multirow{3}{*}{0.85} & \multirow{3}{*}{-} & & & 11 & & \\
\hline & & & & - & $10^{-2}$ & 45 & $10^{-6}$ & 4 \\
\hline & & & & & & 85 & & \\
\hline
\end{tabular}

the radial prefactor $A_{r}$ seems to converge towards unity. These values are close to the expected values of ca. 7.26 and 1 for respectively $A_{N}$ and $A_{r}$ predicted by Eq. 5 and 6 if the scaling factor in Equation 5 were one. However, the data do not extend far enough to confirm this.

We attribute the incomplete convergence of the prefactors (in comparison to the exponents) to two aspects: first, it is a size effect; and we suppose that convergence would improve for larger sheets. The second reason is due to the calculation of the prefactors by fitting a tangent to each data point, whose slope is calculated from the growth exponents. This error is further magnified by the exponential needed to compute the prefactors values.

\section{RESULTS}

The model parameters used to examine the sheet growth algorithm under different scenarios are summarised in Table I. 


\section{A. Sheet growth and layering}

In this section, growth of sheets combined with the aligned layering mechanism is investigated. The ratio $k_{L} / k_{S}$ controls the anisotropy of the structure.

The kinetics parameters are plotted in Fig. 6. The top-left figure shows the evolution of the growth exponent against the logarithm of reduced time, $t k_{S}$. Note, however, that $k_{S}=1$ in all simulations, and $k_{L}$ is the effective free parameter. The apparent difference in reduced start time results from the different length of the initial rate line as $k_{L}$ is varied. The growth exponent increases for all investigated values of $k_{L} / k_{S}$ from about 1 towards a maximum of $\approx 3.5$, before decreasing again towards $\approx 3$.

However, for small values of $k_{L}$, the initial growth is not uniform. In the case of $k_{L} / k_{S}=10^{-7}$, there is a very clear intermediate plateau where $n \approx 2$ between $\ln \left(t k_{S}\right) \approx 2 \ldots 5$. The plateau arises from the temporal separation between layering and sheet growth as $k_{L} / k_{S}$ becomes small. Three-dimensional growth does not commence until $\ln \left(t k_{S}\right) \approx 5$. The delay between reaching a value of 2 and the subsequent increase to 3 depends on the ratio $k_{L} / k_{S}$, which determines the temporal separation between layering and sheet growth.

For small $k_{L}$, in the early stages, the structure is inherently $2 \mathrm{D}$. For large $k_{L}$, the structure is largely $1 \mathrm{D}$ in the very early stages, although, this is not revealed in the data due to poor statistics of small structures. The position of the maximum in the growth exponent is determined by $k_{L} / k_{S}$ - the larger $k_{L}$ is at constant $k_{S}$, the faster the overall process becomes.

The radial growth exponent $n_{r}$ is also plotted as a function of time in Fig. 6 (top-right). For the runs with $k_{L} / k_{S}=10$ and $k_{L} / k_{S}=1$, it increases from values between 0.9 and 0.6 towards about 1.1, and decreases then towards 1 . The maxima of the radial growth exponent occur at the same time as the maxima of the growth exponent. For the simulations with $k_{L} / k_{S}=10^{-3}$ and $k_{L} / k_{S}=10^{-7}$, the behaviour is different. Initially, the radial growth increases towards 1 . Subsequently, it reaches a plateau, although there is a sharp minimum in the plateau. The positions of these minima coincide with the positions of the second increase of the growth exponent in Fig. 6 (top-left).

The fitted prefactors of the power laws $N(t)=A_{N} t^{n}$ and $r_{g}(t)=N_{r} t^{n_{r}}$, as seen in Fig. 6 (bottom row), vary significantly over the simulation time, without reaching a constant value. However, there are significant features in the curves occurring at the same characteristic time as before.

The general behaviour is analogous to a 3D Eden model. The segments form a triangular-prismatic lattice which leads to 3D Eden-like growth with anisotropic growth rates. Thus, the limiting growth exponent approaches 3 , so the characteristic size of the cluster is expected to increase approximately linearly.
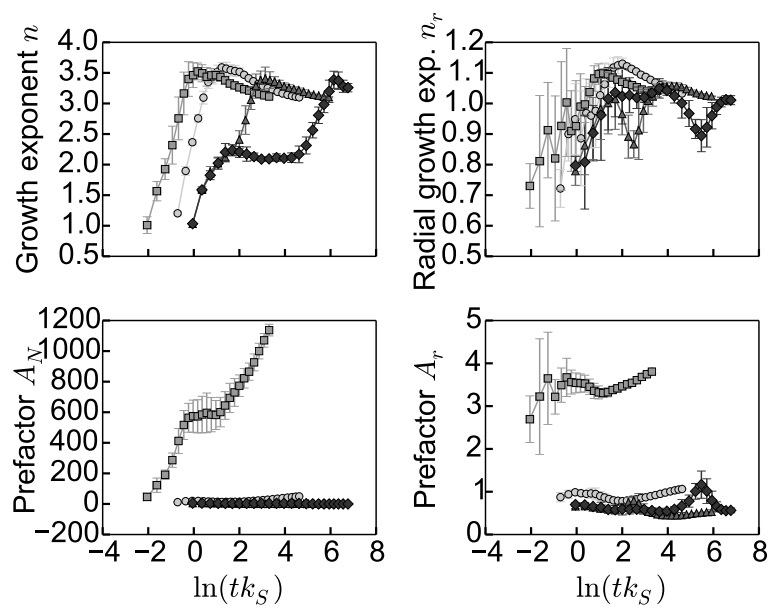

FIG. 6. Kinetic plots for unhindered sheet growth with the layering mechanism. $-k_{L} / k_{S}=10,--k_{L} / k_{S}=1$, $-\Delta-k_{L} / k_{S}=10^{-3},-\downarrow-k_{L} / k_{S}=10^{-7}$ The errorbars are the standard error of the mean after six repetitions.

\section{B. Sheet growth and bifurcation: spherulite-like clusters?}

In this section, sheet growth and bifurcation from a single nucleus is investigated, using the parameters given in Table I. Two bifurcation rates were examined. This is expressed as the group $k_{B} / k_{S}$ being either $10^{-2}$ or $10^{-3}$. Three bifurcation angles were also investigated, $11^{\circ}, 45^{\circ}$ and $85^{\circ}$. Growth was always stopped as soon as a segment intersected with the domain boundary in order to exclude boundary effects. Example structures are shown in Fig. 7. Those clusters grown with $\gamma_{B}=45^{\circ}$ and $\gamma_{B}=90^{\circ}$ are approximately spherical, whilst the cluster grown with $\gamma_{B}=11^{\circ}$ has a doughnut-like shape. The inspection of the cross-sections through the three structures reveals orientational anisotropy in the clusters.

Spherulites are spherical shapes occurring during crystallisation processes, assembled from outwardly-radiating fibres [38]. The structures presented here might be seen as an extension of this concept, where the essentially 1D fibres are replaced by $2 \mathrm{D}$ sheets.

The kinetic data for the growth of clusters with growth and bifurcation is shown in Fig. 8. The first column shows the calculated growth exponents for the two $k_{B} / k_{S}$ ratios investigated, and the second column shows the radial growth exponents calculated from the radius of gyration. The error bars are standard errors of the mean for six repeated simulations.

The growth exponent, presented in the plots of the first column in Fig. 8, takes initial values between 1 and 1.5 and increases subsequently to 2 . If $k_{B} / k_{S}$ is $10^{-2}$, the growth exponents increase more or less directly towards 4 and 4.5. The growth exponents then decrease and seem to approach 3 . If $k_{B} / k_{S}$ is $10^{-3}$, the growth plateaus at 2 for a while, before increasing further towards peak values 


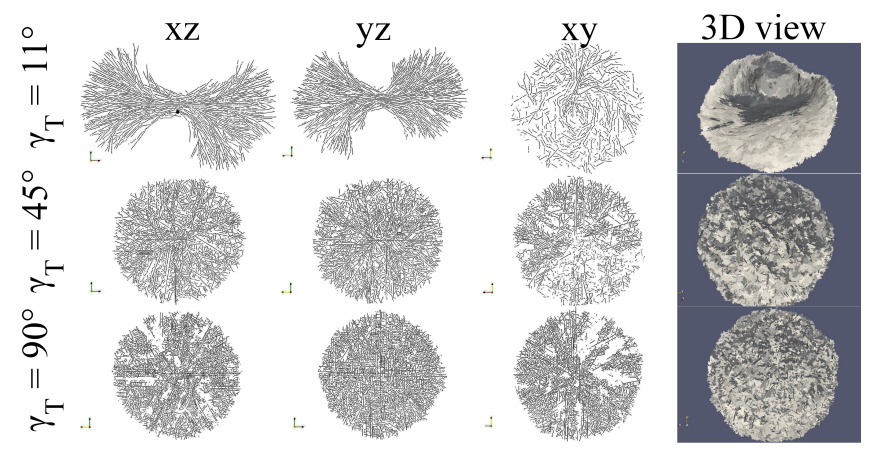

FIG. 7. Cross sections through clusters grown by combining planar sheet growth with bifurcation. The first three columns show different the $y z-, x z$ - and $x y$-cross sections through the clusters. The last column shows a $3 \mathrm{D}$ rendering of the cluster. The first row shows clusters grown with a bifurcation angle $\gamma_{B}=11^{\circ}$, the second with $\gamma_{B}=45^{\circ}$ and the last one with $\gamma_{B}=90^{\circ}$. Note the isotropy of the clusters which becomes apparent by inspection of the different cross sections. The bifurcation rate was always $k_{B}=0.01$. The cross sections shown were obtained on different simulation runs than those used to analyse the kinetic parameters.

between 4 (for the $85^{\circ}$ case) and 5.1 (for the $45^{\circ}$ case). The shape of all curves is similar, which suggests that they may also approach 3 for larger structures. However, since it was not possible to investigate larger structures with the current version of the code, this remains speculative.

The radial growth exponent $n_{r}$ is plotted in the second column of Fig. 8. At short times, there is considerable statistical fluctuation, but notwithstanding, it is apparent that the radial growth exponents first increase toward 1.05. Thereafter, there is indication of decrease towards a minimum followed by a second increase and subsequent second decrease. This indication is strongest for the low bifurcation rate data and for high bifurcation angles. This picture is consistent with the observation for the growth exponents. It is notable that for the models with the lower bifurcation rate, the time to reach the maximum seems to be less dependent on the bifurcation angle $\gamma_{B}$ than for those models with high bifurcation rate.

The observed growth exponents between 4 and 5 are in the range that is predicted by Eq. 10 if a second generation of bifurcation sheets is formed before initial impingement. From this point on, the growth exponent decreases. The results suggest that the growth exponent will eventually approach 3 . This is consistent with Leyvraz's observations, that eventually only those growth sites at the surface of the convex hull can sustain further growth [30].
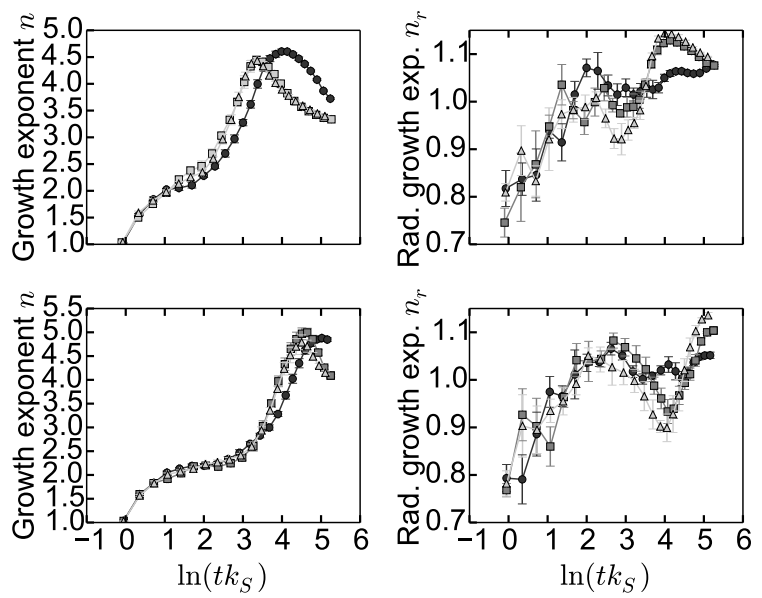

FIG. 8. Growth exponents $n$ and radial growth exponent $n_{r}$ for unhindered sheet growth with bifurcation. The top row shows the simulations with $k_{B} / k_{S}=10^{-2}$, the bottom row with $k_{B} / k_{S}=10^{-3}$. For both $k_{B} / k_{S}$-ratios, three bifurcation angles were investigated: $-\bullet-\gamma_{B}=11^{\circ},-\square-\gamma_{B}=45^{\circ}$, $-\triangle-\gamma_{B}=85^{\circ}$. The error bars represent the standard error of the mean for six repetitions and may be smaller than the dots.

\section{Space filling by growing sheets with continuous nucleation}

In this section, the results for the model of space filling by sheet growth with continuous nucleation (Fig. 2b) are presented. The structures were grown in a cubic domain with periodic boundary conditions. Exemplar cross sections are shown in Fig. 9. The left column shows structures evolved to about $50 \%$ of the final solid volume fraction, and the right column shows the final structures. From top to bottom, the bulk nucleation rate increases with details given in Table I. Pockets of empty space, bound by sheets, are formed. These are of polygonal shape in the cross section, indicating that they are polyhedra in 3D space. As the structures evolve, the pockets are filled by smaller sheets.

Figure 10 shows growth exponents derived from the Avrami plot as a function of simulation time, averaged over multiple simulations. In this Figure, time is normalised by $t^{*}$, the characteristic time for the onset of blocking (Eq. 3) rather than $k_{S}$. The growth exponent exhibits three distinct regimes: the initial growth exponent is approximately 0.6 and increases quickly to a value of slightly larger than 3 . It remains approximately constant for a while, followed by a steep decrease, after which the growth exponent increases again to a value close to 0.3 , following which it decreases slowly. The maximum value of the exponent in this third regime seems to be weakly dependent on the nucleation rate, being close to 0.2 for those runs with higher, and approaching 0.3 for those with lower nucleation rate.

The initial behaviour of the model is due to the undis- 
turbed growth kinetics of individual sheets. In Sec. III A, it was shown how sheets evolving from nuclei start with low growth exponents, which increase towards values slightly in excess of 2 and then decrease slowly. This is the picture observed here, but the growth exponent takes a value of $3(2+1)$ due to the continuous nucleation regime. The sharp drop in the growth exponent is caused by the impingement of the sheets.

The regime following the steep decay of the growth exponent is the blocking regime according to Kooi [27]. Equation 3 suggests a growth exponent of $1 / 3$ in this regime in the long time limit. This value is not reached in any of the runs presented. Instead, the maximum value of the growth exponent in the blocking regime remains lower at about 0.3 . This is probably caused by the finite size of the nuclei, whilst Kooi's theory assumes nucleation of infinitesimal small nuclei. As the structures evolve and the free spaces between the pockets of sheets fill up, fewer nuclei can be inserted, which decreases the growth exponent. In addition to the finite simulation size effect, it is also noted that anisotropy of the nuclei can also have an effect on the growth rate. This is the case when the sheets are very thin, such that the nucleus-segments can only be inserted aligned to the sheets if they are inserted close to a surface of the already grown structure. This shows that the on-lattice model with limited-orientation presented by Kooi [27] captures the essential physics of the more general case with arbitrary orientations that is presented here. It is interesting to note that the cross sections shown in Figure 9 exhibit similartiy to snapshots of simulations for the case of $1 \mathrm{D}$ growth in a $2 \mathrm{D}$ domain $[10,11]$. The problems studied by these authors belong to the same class of problem as the work studied in this contribution; $\left(d_{g}\right)$-dimensional growth within a $d_{g}+1$ dimensional space. An interesting subject for future work is the verification of the scaling law for the volume of cells given by Krapivsky and Ben-Naim for 2D growth in 3D [11] and the extension of the mean-field theory of Hwang and Eryilmaz to 2D growth in 3D [10].

\section{Sheet growth and layering: space filling by sheet crystals}

In this section, space filling by growing sheet crystals is studied. Sheet crystals form if the sheet growth and layering mechanisms are active in a continuous nucleation scenario, as discussed in Sec. IV A. The parameters for the simulations are shown in Table I.

The first column of Fig. 11, reports cross sections through structures evolved to about $50 \%$ of the final solid volume fraction. The second column shows the final structures. The ratio $k_{L} / k_{S}$ decreases from the top to the bottom. At high $k_{L} / k_{S}$ the clusters grow in the shape of a cigar-shaped ellipsoid. This transitions via a sphere towards a flat oblate ellipsoid as $k_{L} / k_{S}$ decreases. The intermediate structure shown in Fig. 11g has only a few layers of repetition, indicating that the layering rate
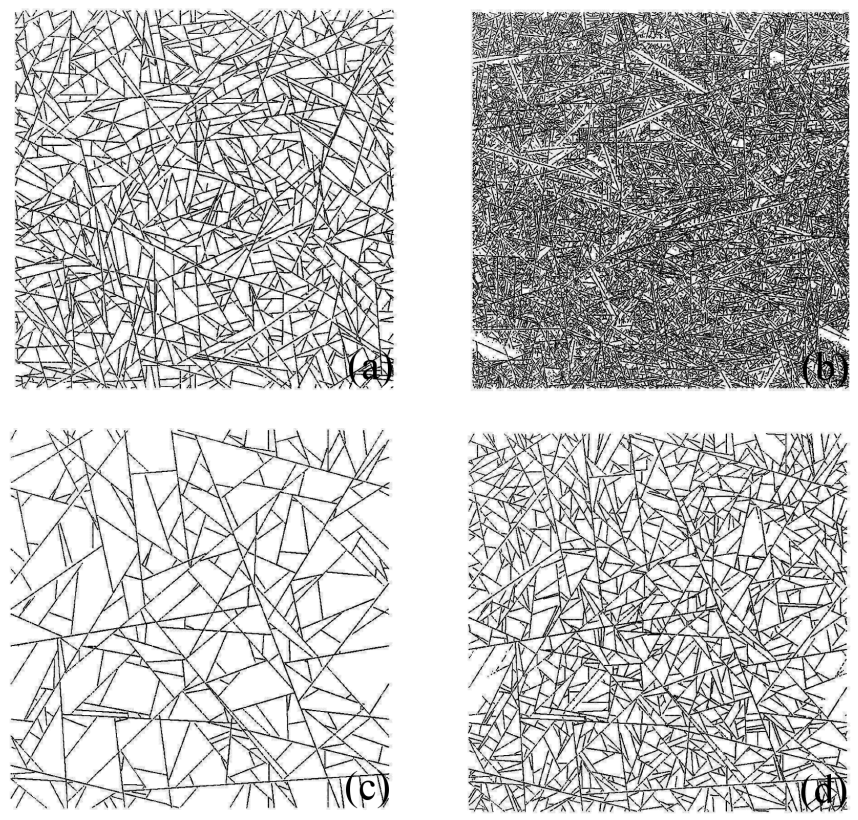

FIG. 9. Cross sections through structures grown for sheet growth with continuous nucleation. Left column contains structures evolved to approximately $50 \%$ of the final solid volume fraction. Right column: structures at the end of the simulation. Top: $k_{N} / k_{S}=10^{-6}$, bottom: $k_{N} / k_{S}=10^{-8}$.

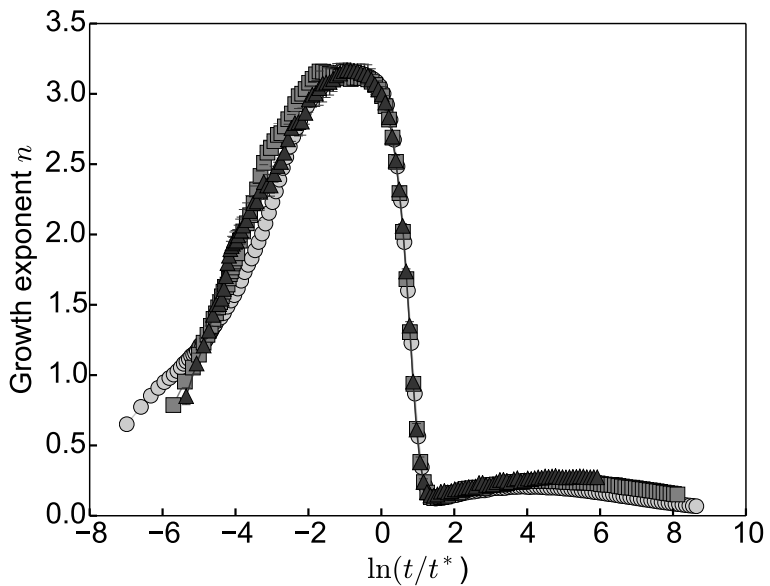

FIG. 10. Growth exponent plot for space filling by sheets. Each line corresponds to slightly different kinetic parameters: $-k_{N} / k_{S}=10^{-6},-\square-k_{N} / k_{S}=10^{-7},-\boldsymbol{\Delta}-k_{N} / k_{S}=$ $10^{-8}$. The error bars represent the standard error of the mean of four repetitions.

is so low that it only plays a minor role compared to nucleation and sheet growth. The structure appears similar to those shown for the sheet growth and continuous nucleation scenario shown in Fig. 9.

The kinetic data are shown in Fig. 12. The time dependence of the growth exponent depends strongly on $k_{L} / k_{S}$. In the case of $k_{L} / k_{S}=1$ and $k_{L} / k_{S}=10$, the 
growth exponent starts close to 1 and increases towards a value close to 4.5 . They then slowly decreases to about 4.2. Thereafter, there is a steep decrease, after which the evolution of the structure terminates.

The growth exponent observed for the models with $k_{L} / k_{S}=10^{-3}$ and $10^{-4}$ increases towards 3 , seems to plateau a short while and then increases towards 4 . This is followed by a sharp drop, either towards 0 for $k_{L} / k_{S}=10^{-3}$ or towards a shoulder at about 1 , before dropping to 0 . If the layering is much slower than the sheet growth, $k_{L} / k_{S}=10^{-7}$, the maximum growth exponent is only slightly larger than 3 . After staying at about 3 for a short while, it drops towards a value of approximately 0.3 and decreases very slowly. The curve appears to be very similar to those shown in Fig. 10. The time scale of layer formation is smaller than that of impingement, thus, supporting the observations made previously about Fig. 11.

The different behaviour of the models is caused by the separation of the time-scales between sheet growth and layering and hence the resultant anisotropy of the growing shapes. If the ratio is close to 1 for the chosen sheet height $h$ and spacing $d$, the growing clusters have only mild anisotropy and therefore, the KJMA equation (Eq. 1) applies. The initial behaviour for small clusters is always due to the initial stabilisation of the growth kinetics and is similar to the observations in Sec. IV A, where it can be seen that the growth exponent of the cluster volume reaches 3.5 before it approaches 3 . Thus, in the continuous nucleation scenario, the peak value of the growth exponent reaches 4.5. The sharp decay of the growth rate is due to impingement of growth from the different clusters. This deviation from the KJMA equation is caused by impingement. A small gap remains between impinging clusters due to the finite size of the inserted triangles. These gaps are about $5 \%$ of the volume of the final structures for the simulations shown in this section. The exponential in the KJMA equation is highly sensitive to this detail, which causes the sharp drop in the growth exponent at the end of the simulations.

In the simulation with $k_{L} / k_{S}=10^{-3}$, the time scale for sheet growth is short compared to the time scale of layering due to the small value of $k_{L} / k_{S}$. It is constructive to compare with the equivalent situation for growth from a single nucleus shown in Fig. 6. In the former case, growth becomes hindered, in the latter case, it remains unhindered. Initially, in the multiple-nucleation site case, the growth exponent reaches a value of about $3(2+1$ since it is $2 \mathrm{D}$ growth and nucleation) and the model behaves like a pure sheet growth model, like the single nucleation site model. As the layering starts playing a role in the multiple nucleation case, the growth exponent increases to four $(3+1$ since it is now $3 \mathrm{D}$ growth and nucleation). When the sheets start to impinge, the growth exponent decreases rapidly. On the other hand, in the single nucleus case, the growth exponent only reaches three $(3+0)$, and there is no decrease since there is no impingement. The same applies to the run with $k_{L} / k_{S}=10^{-4}$. As
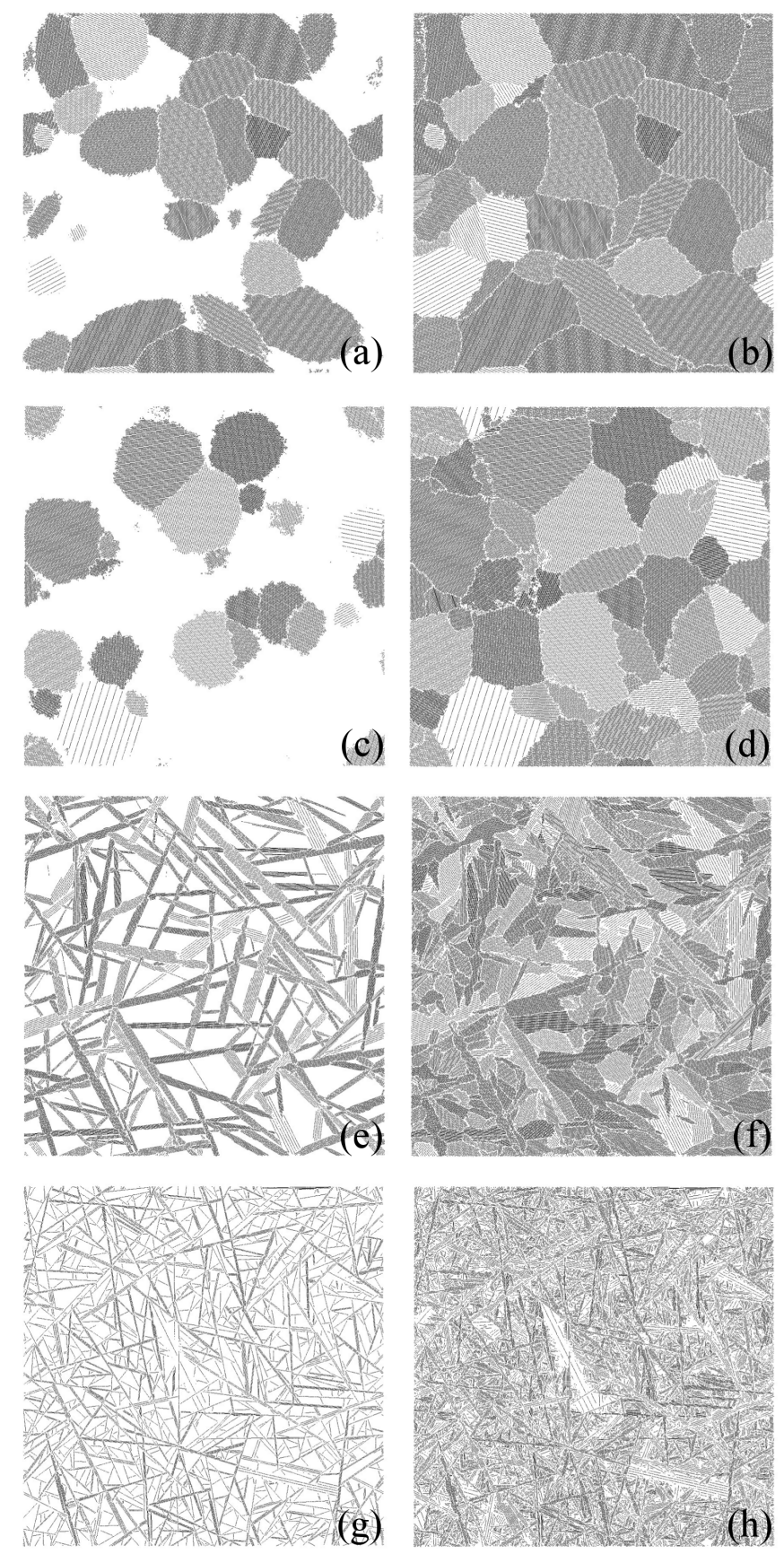

FIG. 11. Example structures for space filling by sheet crystals. First column: ca. $50 \%$ evolved, second column: final structures. Top to bottom: $k_{L} / k_{S}=10, k_{L} / k_{S}=1, k_{L} / k_{S}=10^{-4}$ and $k_{L} / k_{S}=10^{-7}$.

a final note, the shoulder in the decrease of the growth exponent in this run in the multiple nucleation site case with a value of 1 , seen in Fig. 11, is due to the quasi-1D filling-in of the voids seen in Fig. 11e, by the layering mechanism. A similar feature can also be seen in the works of Pusztai et al. and Shepilov and Baik [20, 25] for growth of ellipsoids.

In the model with $k_{L} / k_{S}=10^{-7}$, the time scales for 
growth and layering are so different that layering only plays a minor role prior to impingement. Therefore, the growth exponent barely exceeds 3 (2D growth + nucleation), which shows that the model behaves like a model of impinging sheets. The influence of layering after impingement also remains negligible, since the curve has the same shape as pure sheet growth with continuous nucleation. Therefore, the structure cross sections appear to be similar to those shown in Fig. 10.

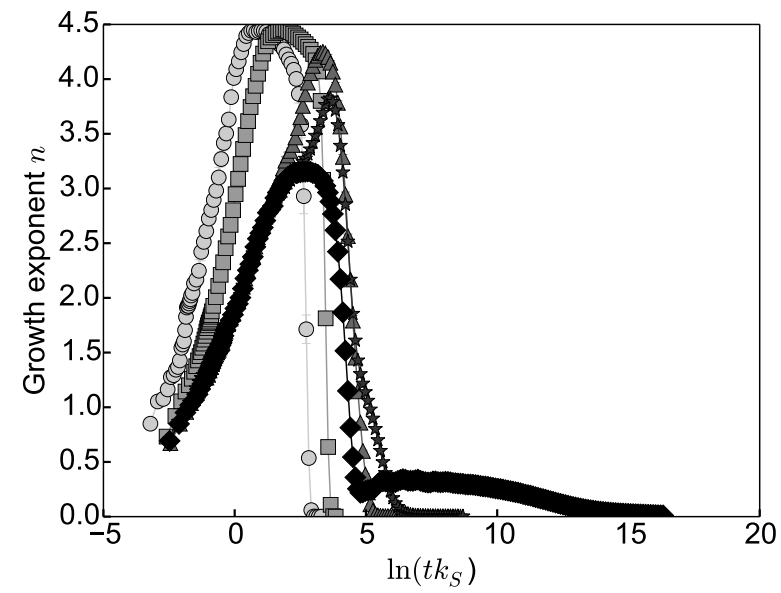

FIG. 12. Derived growth exponents for space filling by sheet growth and layering. $-k_{L} / k_{S}=10,-\square-k_{N} / k_{S}=1$, $-\Delta-k_{N} / k_{S}=10^{-3},-\star-k_{N} / k_{S}=10^{-4},--k_{N} / k_{S}=$ $10^{-7}$. The error bars denote the standard error of the mean of four repetitions.

\section{E. Space filling by sheet growth with bifurcation}

In this section, space filling is investigated in a continuous nucleation scenario, with clusters growing by the sheet growth and bifurcation mechanisms. The parameters are given in Table I.

In Fig. 13, cross sections through intermediate structures are shown in the first column and evolved structures in the second. The changing parameters between the rows is the bifurcation angle $\gamma_{B}$, which take the values $11^{\circ}, 45^{\circ}$ and $85^{\circ}$. Fig. 13a shows elongated packets of sheets, Fig. 13c and 13e show more spherical clusters. This agrees well with the observations made in Sec. IV B. The fully evolved structures are dense and disordered. The density appears to be homogeneous.

The growth exponent as a function of time is shown in Fig. 14 and appear largely independent of the bifurcation angle. The initial growth exponent is about 1 and increases towards a maximum of about 5. Subsequently, the growth rate drops sharply and the evolution of the structure terminates.

The initial increase of the growth exponent is following the undisturbed cluster growth kinetics, which has
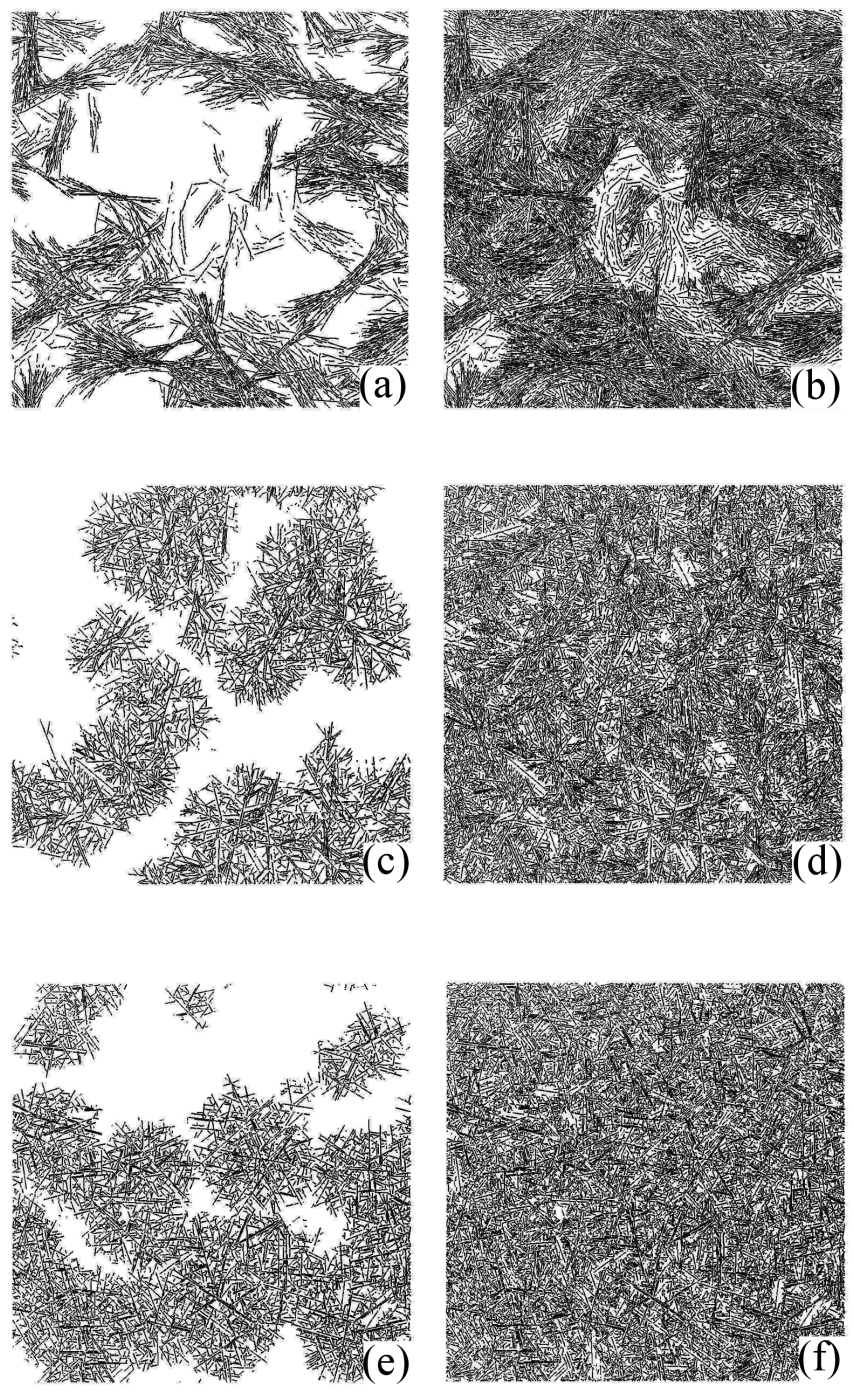

FIG. 13. Example structures for space filling by sheet growth and bifurcation. First column: ca. $50 \%$ evolved, second column: final structures. In all cases $k_{B} / k_{S}=10^{-2}$. From top to bottom: $\gamma_{B}=11^{\circ}, \gamma_{B}=45^{\circ}$ and $\gamma_{B}=85^{\circ}$.

already been studied in Sec. IV B. The maximum value of the growth exponent reaches about 5.5, before dropping sharply. This indicates that impingement between different clusters becomes important before the growth exponent decreases due to the internal impingement of the sheets formed by bifurcation, more or less independent of the bifurcation angle, as found in Sec. IV B.

The onset of the sharp decrease of the growth exponent indicates that the clusters have started impinging. The sharp decay itself indicates that little ingrowth between the individual clusters occurs. The long time period with a near-to-zero growth exponent is due to the densification of the structure by ongoing nucleation events within the pore space of the clusters. This is a similar behaviour to the dense sheet crystals discussed in Sec. IV D. Thus, the kinetic behaviour of these structures might be approximated by an KJMA style model, if the radial growth rate 


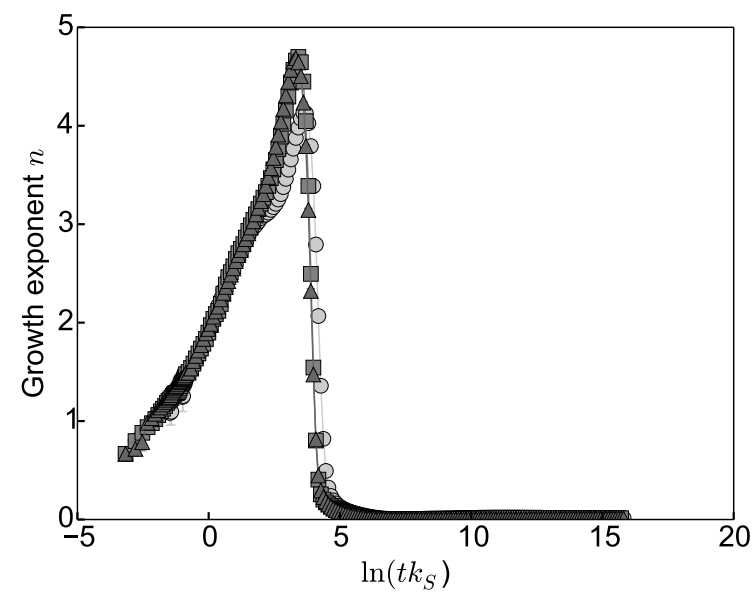

FIG. 14. Growth exponent for space filling by sheet growth and bifurcation in a continuous nucleation scenario. $k_{B} / k_{S}=$ $10^{-2}$, the three investigated angles were $-\bullet-\gamma_{B}=11^{\circ},-\square-$ $\gamma_{B}=45^{\circ},-\boldsymbol{\Delta}-\gamma_{B}=85^{\circ}$.

is calibrated accordingly.

\section{DISCUSSION AND CONCLUSION}

In this paper, a method of $3 \mathrm{D}$ space filling by the growth of $2 \mathrm{D}$ sheets is introduced and the resultant structures were studied. These studies were only possible by introducing an off-lattice kinetic Monte Carlo model to describe the growth. Different mechanisms, such as layering and bifurcation, are proposed to allow a 2D growth mechanism to fill 3D space. These mechanisms can be combined in a variety of subtly different models that lead to substantially different structures. Layering leads to highly-ordered structures whereas bifurcation creates amorphous structures.

Our model explores the consequences of twodimensional growth in three-dimensional domains, regardless of the details of the underlying growth mechanisms. This becomes apparent if one compares our analysis to other models such as the particle aggregation model with collective cluster moves described by Whitelam and Geissler [39].

Whitelam and Geissler study the aggregation of particles to quasi-2D clusters due to an interaction potential which leads to sticky particles around the equator. Particle aggregation is reversible, and the particle clusters are able to do diffusive movements in the domain.

Our analysis presumes a situation, in which 2D growth is observed as the net-phenomenon, so our analysis could be seen as a coarse grained version of Whitelam and Geissler's work. Our results apply equally to a situation where (non-colloid) crystals precipitate from solution, where the quasi-2D shape is controlled by the arrangement of atoms within the elementary cell.
Whitelam and Geissler's colloidal growth mechanism raises three important points for future work. Many growth processes are locally reversible and depend on the availability of nutrients, which can be dissolved species (crystal growth), colloidal particles in the case of colloidal aggregation or other substances required to sustain growth. The aforementioned authors treat explicitly by tracking the particles within the domain. We think that an extension of our model to include nutrient transport through the grown structure is of great interest, since sheets can easily create subspaces which are at least poorly connected to the remaining domain, where nutrients could quickly become scarce. This has also been proposed for the application which provided our starting point [3]. Furthermore, the clusters in Whitelams and Geissler's model are mobile, something which might have an effect on the final geometry, which could also be an interesting subject for further work.

It is shown that the model consisting of sheet growth and layering leads to layered sheet crystals, whose aspect ratio depends on the ratio between the two processes. In a continuous nucleation scenario, this leads to polycrystalline structures. However, the growth kinetics depend on the ratio between layering and nucleation rates: if the layering rate is much slower the model behaves like a sheet-growth model, if it much faster it behaves similarly to a model of $3 \mathrm{D}$ anisotropic growing structures.

The combination of sheet growth and the bifurcation mechanism leads to 3D structures resembling spherulites but with an almost amorphous internal sheet structure instead of needles. The kinetics for these models evolve differently at different time scales. Initially, low growth exponents are observed, which increase towards a maximum. We have presented arguments as to why the maximum growth exponents are expected to lie between 4.0 and 6.0 as observed in the simulations. Beyond the maximum, the growth exponents appears to decay towards a value of 3 , which is in agreement with theoretical arguments. Space filling by clusters grown with the bifurcation mechanism exhibits high growth exponents and a sharp drop in the growth rate. Besides the values of the growth exponents, the curves resemble those of impingement of sheet crystals. Thus, the clusters cannot grow into each other but growth stops at collision.

The clusters emerging from the bifurcation model can have (if $k_{B} / k_{S}$ is large) an open internal structure, if the sheet growth rate is large compared to the bifurcation rate. Various authors (for example Bishnoi and Scrivener, Scherer et al $[40,41])$ proposed the rapid formation of an open hydration product in cement, which may densify as the hardening progresses. Growth of the structures according to the bifurcation model leads to space-filling by spherulite-like open structures, which could then densify using a slow layering step.

The computer model presented in this paper only handles full triangles. Any impingement of sheets always leads to the rejection of those triangles which intersect with any previously grown sheet. Also, nuclei are of finite 
size. Therefore, in this model, space can never be totally filled. We do not expect that this has any qualitative effect on the observed kinetics other than those mentioned previously. However, it has a profound effect on some of the properties of the structures grown. Whilst the effect on the spatial arrangement is rather subtle, one expects the gaps to have a huge effect on structural properties like the permeability.

This is the subject of ongoing work by these authors, which revolves around an adaptive segment shape algorithm, which allows the segment shape to altered, to close the remaining gaps between the sheets.

The model presented in the paper is capable of reproducing a large range of crystal-structure types with relatively few model parameters. This provides the prospect of designing material model structures for a host of appli- cations with individually-tuned properties, for example, for porosity, density, and surface-to-volume ratio, which could then be exploited for second-stage modelling such as of reactive transport within such a structure.

\section{ACKNOWLEDGMENTS}

This research was supported by the European Union Seventh Framework Programme (FP7/2007-2013) under grant agreement 264448. AFR was supported by EPSRC grant EP/H035397/1. MAE would like to thank T. Michelsen (Oldenburg) for his advice regarding the algorithm.
[1] M. A. Etzold, P. J. McDonald, and A. F. Routh, Cem. Conc. Res. 63, 137 (2014).

[2] R. F. Feldman and P. J. Sereda, Eng. J. - Canada 53, $53(1970)$.

[3] J. W. Bullard, H. M. Jennings, R. A. Livingston, A. Nonat, G. W. Scherer, J. S. Schweitzer, K. L. Scrivener, and J. J. Thomas, Cem. Conc. Res. 41, 1208 (2011).

[4] K. L. Scrivener and R. J. Kirkpatrick, Cem. Conc. Res. 38, 128 (2008).

[5] E. M. Gartner, Cem. Conc. Res. 27, 665 (1997).

[6] M. J. Blunt, B. Bijeljic, H. Dong, O. Gharbi, S. Iglauer, P. Mostaghimi, A. Paluszny, and C. Pentland, Advances in Water Resources 51, 197 (2013).

[7] S. V. Churakov and T. Gimmi, J. Phys.Chem. C 115, 6703 (2011).

[8] W. Ding, H. Li, P. Pfeifer, and R. Dittmeyer, Chem. Eng. J. (2014).

[9] M. B. Holness, Contrib. Mineral. Petr. 168 (2014).

[10] W. Hwang and E. Eryilmaz, Phys. Rev. Lett. 113, 025502 (2014).

[11] P. L. Krapivsky and E. Ben-Naim, Phys. Rev. E 50, 3502 (1994).

[12] A. Kolmogorov, Izv. Akad. Nauk SSR. Ser. Mat. , 367 (1937).

[13] W. A. Johnson and R. F. Mehl, Trans. AIME 135, 416 (1939).

[14] M. Avrami, J. Chem. Phys. 8, 212 (1940).

[15] K. Sekimoto, Physica A 135, 328 (1986).

[16] E. Ben-Naim and P. L. Krapivsky, Phys. Rev. E 54, 3562 (1996).

[17] M. R. Riedel and S.-i. Karato, Geophys. J. Int 125, 397 (1996).

[18] J. Farjas and P. Roura, Phys. Rev. B 75, 184112 (2007).

[19] J. Farjas and P. Roura, Phys. Rev. B 78, 144101 (2008).

[20] M. P. Shepilov and D. S. Baik, J. Non.-Cryst. Solids 171, 141 (1994).

[21] N. V. Alekseechkin, J. Non.-Cryst. Solids 357, 3159 (2011).

[22] M. Tomellini and M. Fanfoni, Phys. Rev. E 85, 021606
(2012).

[23] B. A. Berg and S. Dubey, Phys. Rev. Lett. 100, 165702 (2008).

[24] E. VIlla and P. R. Rios, Acta Mater. 58, 2752 (2010).

[25] T. Pusztai and L. Gránásy, Phys. Rev. B 57, 14110 (1998).

[26] R. B. Godiksen, P. R. Rios, R. A. Vandermeer, S. Schmidt, and D. J. Jensen, Scripta Mater. 2008, 279 (58).

[27] B. J. Kooi, Phys. Rev. B 73, 054103 (2006).

[28] J. Neyman, ed., A two-dimensional growth process, Proceedings of the Fourth Berkely Symposium on Mathematical Statistics and Probability, Vol. IV Biology and problems of health (1961).

[29] H. Peters, D. Stauffer, H. P. Hölters, and K. Loewenich, Z. Physik B 34, 399 (1979).

[30] F. Leyvraz, J. Phys. A-Math. Gen. 18, L941 (1985).

[31] "CGAL, Computational Geometry Algorithms Library," Http://www.cgal.org.

[32] A. B. Bortz, M. H. Kalos, and J. L. Lebowitz, J. Comput. Phys. 17, 10 (1975).

[33] D. T. Gillespie, J. Comp. Phys. 22, 403 (1976).

[34] G. H. Gilmer, J. Cryst. Growth 35, 15 (1976).

[35] A. Chatterjee and D. G. Vlachos, J. Computer-Adided Mater. Des. 14, 253 (2007).

[36] A. Voter, in Radiation Effects in Solids (Springer, NATO Publishing Unit, 2005).

[37] M. A. Novotny, "A Tutorial on Advanced Dynamic Monte Carlo Methods for Systems with Discrete State Spaces," (World Scientific, 2001) Chap. 3, pp. 153-210.

[38] L. L. L.Gránásy, A. Szállás, B. Korbuly, G. I. Tóth, L. Környei, and T. Pusztai, Metallurgical and Materials Transactions A: Physical Metallurgy and Materials Science 45A, 1695 (2014).

[39] S. Whitelam and P. L. Geissler, J. Chem. Phys. 127, 154101 (2007).

[40] S. Bishnoi and K. Scrivener, Cem. Conc. Res. 39, 849 (2009).

[41] G. W. Scherer, J. Zhang, and J. J. Thomas, Cem. Conc. Res. 42, 982 (2012). 